Article

\title{
An Updated Constraint on Variations of the Fine-Structure Constant Using Wavelengths of Fe II Absorption Line Multiplets
}

\author{
T. D. Le L,2 $^{1, D}$ \\ 1 Division of Computational Physics, Institute for Computational Science, Ton Duc Thang University, \\ Ho Chi Minh City 700000, Vietnam; leducthong@tdtu.edu.vn \\ 2 Faculty of Applied Sciences, Ton Duc Thang University, Ho Chi Minh City 700000, Vietnam
}

Received: 6 November 2018; Accepted: 3 December 2018; Published: 5 December 2018

\begin{abstract}
A new stringent limit relating to the variation of the fine-structure constant $\left(\alpha=\frac{e^{2}}{4 \pi \varepsilon_{0} \hbar c}\right)$ has been extracted from Ritz wavelengths of 27 quasi_stellar object (QSO) absorption spectra lines of Fe II. The calculation was combined with laboratory wavelengths and QSO spectra to obtain the result $\Delta \alpha / \alpha=(0.027 \pm 0.832) \times 10^{-6}$. This result suggests how dedicated astrophysical estimations can improve these limits in the future and can also constrain space_time variations.
\end{abstract}

Keywords: varying constants; varying fine-structure constant; Ritz wavelengths; absorption spectra analysis

\section{Introduction}

One of the most interesting problems of contemporary physics addresses space_time variations of the fundamental constants across the evolution of the Universe, a possibility considered by Dirac [1] and Milne [2]. One possible variation indicated by measurements of QSO emission spectra suggests that the fine-structure constant, $\alpha$, may have a changing value throughout the evolution of the Universe [3,4]. From the analysis of the spectra, some of the achievements of the last years have already been recited by the alkali doublet (AD) method. The most suitable result of this method is $\Delta \alpha / \alpha=(0.7 \pm 1.4) \times 10^{-4}$ with the redshifts $0.16<z<0.80$ and $\Delta \alpha / \alpha=(1.5 \pm 0.7) \times 10^{-3}$ up to redshift $0.281[5,6]$ derived from the analysis of the wavelength separations of O III emission alkali doublet lines $(\lambda 5007 \AA$ and $\lambda 4959 \AA)$ seen in the Sloan Digital Sky Survey ( SDSS) QSO. The other method is called the many multiplet (MM) method, which obtained the changed values of $\Delta \alpha / \alpha$, an order sensitive magnitude better than the AD method [7-10]. Murphy et al. (2003) applied this technique to analyze the $21 \mathrm{Si}$ IV doublets, resulting in $\Delta \alpha / \alpha=(-0.5 \pm 1.3) \times 10^{-5}$ at $z_{a b s}=2.8$ [11]. Furthermore, within the first development of these studies, Dzuba et al. $(1999 ; 2003)$ applied this method to several different transitions of multiplets and ions in order to exploit the sensitivities of $\alpha$-variation [7-10]. Applying the MM method to 128 systems with the spanning redshift $0.2<z<3.7$, Murphy et al. (2002) and Webb et al. (2003) obtained $\Delta \alpha / \alpha=(-0.57 \pm 0.10) \times 10^{-5}[12,13]$. Moreover, the application of this approach used large numbers of Echelle quasar spectra with a good resolving power, attempting to find the cosmological variations in $\alpha$. Reimers et al. (1998) and Quast et al. (2004) obtained $\Delta \alpha / \alpha=(-0.4 \pm 1.9) \times 10^{-6}[14,15]$, while Chand et al. (2006) determined $\Delta \alpha / \alpha=(-0.5 \pm 2.4) \times 10^{-6}$ [16]. However, the estimation errors of $\Delta \alpha / \alpha$ are going to be quite challenging within the determinations of true values when applying the MM technique to large samples, because the wavelength calibration errors have not been taken into account. The most accurate estimate of $\Delta \alpha / \alpha$ gave the space or time variation of $\Delta \alpha / \alpha$ with high accuracy (parts of $2 \times 10^{-6}$ ) in order to apply the MM method to Fe II absorption lines at $z_{a b s}$ $=1.15$, in which Levshakov et al. $(2006 ; 2007 ; 2008)$ obtained $\Delta \alpha / \alpha=(-0.07 \pm 0.84) \times 10^{-6}[17-19]$, 
and Porsev et al. (2007) found $\Delta \alpha / \alpha=(-0.12 \pm 1.79) \times 10^{-6}$ and $\Delta \alpha / \alpha=(-5.4 \pm 2.5) \times 10^{-6}$ at $z=1.8$ [20]. These studies reflect the possibility that the ultimate resulting estimation of $\Delta \alpha / \alpha$ might be affected by unnoticed systematic effects that are included and correlated with the individual values from different pairs. In combination with wavelength calibration difficulties, problems with the methodology might be the cause when this is applied towards the analysis of the Fe II absorption complex and could lead to different sensitivity contributions to variations of $\alpha$ from the fact that different transitions were found in molecules and atoms from QSO spectra [21-27]. Following on this interesting topic, a new method has been developed and achieved to search for $\alpha$-variation over cosmological space and time [28]. The obtained results inferred a change with the cosmic epoch of $\Delta \alpha / \alpha=(-0.157 \pm 0.300) \times 10^{-6}$ together with the redshift $z=1.15$ [28]. This technique allowed us to estimate values at the first stages of the evolution of the universe through the exploration of both doublet and multiplet lines visible in QSO spectra. This method has the benefit of being more obvious and less affected by the systematic error.

In this study, we propose to make use of the Ritz wavelengths of 27 QSO absorption line spectra of Fe II to constrain any variations in the fine-structure constant on cosmological space and time scales [29-33]. Combining high precision measurements of the light from distant quasars together with laboratory wavelengths of the measured lines, this result is significantly tighter than the previously derived results using the MM method [34].

\section{Atomic Data and Analysis}

The absorption lines observed inside of the spectra of quasars offer us an effective tool to constrain changes in the fine-structure constant. They include almost-resonance lines belonging to the ions, as well as the ground state transition of those correspondences. The separation between levels of energy as a consequence of fine-splitting is proportional to $\alpha^{4}$, with the main term of the energy level being proportional to $\alpha^{2}$. Any difference in these separations could possibly be due to a change in $\alpha$, because the energy associated with the line transition depends on individual changes. The spatial or temporal variation of the fine-structure constant can create a difference throughout the measurement relationship between the QSO absorption and emission systems and the laboratory values [28].

$$
\frac{\Delta \alpha}{\alpha} \approx \frac{1}{2}\left(\frac{\frac{1}{2}\left(\frac{\lambda_{2}(t)}{\lambda_{1}(t)}\right)-1}{\frac{1}{2}\left(\frac{\lambda_{2}(0)}{\lambda_{1}(0)}\right)-1}-1\right)
$$

Thus, comparing the relative of the measured wavelengths $\lambda_{1}(t)$ and $\lambda_{2}(t)$ from QSO absorption or emission systems at the redshift $\mathrm{z}$ with the related laboratory wavelength it is possible to directly infer the potential variation at different epochs and space and time locations. The main advantage of this technique is that it is useful for studying the possible effect of temporal variability on the fine-structure constant, comparing its current value with earlier comic space and time or different regions of the universe. This method has a great benefit in that it allows us not only to apply it to the emission lines, but also to the absorption lines, as well as into wide ranges of redshifts. The spectra employed in this particular study are the same as those presented in References [29,32,33], and a detailed procedure of the analysis together with Ritz laboratory wavelengths are presented in the works of Nave and Sansoneti (2011) [30] and Nave (2012) [31]. The details of the experiments with a discussion arising in the literature about the validity of the analysis are available in the papers [27-29]. The spectra under study are of great quality within the uncertainty of laboratory errors of the $\mathrm{Fe}$ II, whose measurements are known up to several $m A$. Making use of this uncertainty allows us to estimate the systematic error in order to determine the value of $\Delta \alpha / \alpha$. In this way, we can apply the Fe II elements and the quality of those ionization states and the original majority of the multiplet lines with the various upper levels of energy for our analysis, with independent physical conditions of the gas where the Fe II lines originate. The possible cause of the statistical and systematic error discussion details can be found in References [15-30]. 
In our study, we used the updated data that were recorded by the Hubble Space Telescope Imaging Spectrograph with the high-resolution far-UV spectrum from the works of Aldenius et al. (2006); Nave and Sansonetti (2011); Nave (2012); and Pickering et al. (2000; 2002) [29-33]. The most suitable spectra, which provide the necessary wavelength accuracy for our study, are presented in Table 1.

Table 1. Twenty-seven quasi-stellar object (QSO) absorption lines of Fe II and Ritz laboratory wavelengths. The lower level for all lines is $3 d^{6}\left({ }^{5} \mathrm{D}\right) 4 \mathrm{sa}^{6} \mathrm{D}_{9 / 2}$.

\begin{tabular}{|c|c|c|c|}
\hline Upper Level & $\begin{array}{l}\text { Observed Wavelengths } \\
\text { from QSO }\end{array}$ & $\begin{array}{c}\text { Ritz Wavelengths from } \\
\text { Laboratories }\end{array}$ & References \\
\hline $3 d^{6}\left({ }^{5} z D\right) 4 p^{6} D_{9 / 2}$ & 2600.172 & $2600.17210(11)$ & [28-30] \\
\hline $3 d^{6}\left({ }^{5} z D\right) 4 p^{6} D_{7 / 2}$ & 2586.649 & 2586.64934(11) & {$[28-30]$} \\
\hline $3 d^{6}\left({ }^{5} z D\right) 4 p^{6} F_{11 / 2}$ & 2382.764 & 2382.76397(11) & {$[28-30]$} \\
\hline $3 d^{6}\left({ }^{5} z D\right) 4 p^{6} F_{9 / 2}$ & 2374.460 & $2374.46009(11)$ & [28-30] \\
\hline $3 d^{6}\left({ }^{5} z D\right) 4 p^{6} F_{7 / 2}$ & 2367.589 & 2367.58924(11) & {$[28-30]$} \\
\hline $3 d^{6}\left({ }^{5} z D\right) 4 p^{6} P_{7 / 2}$ & 2344.212 & 2344.21276(11) & [28-30] \\
\hline $3 d^{6}\left({ }^{5} z D\right) 4 p^{4} F_{9 / 2}$ & 2260.780 & $2260.77911(10)$ & [28-30] \\
\hline $3 d^{6}\left({ }^{5} z D\right) 4 p^{4} D_{7 / 2}$ & 2249.877 & $2249.87547(10)$ & {$[28-30]$} \\
\hline $3 d^{6}\left(a^{3} y F\right) 4 p^{4} F_{7 / 2}$ & 1611.200 & 1611.20037(7) & {$[28-31]$} \\
\hline $3 d^{5}\left({ }^{6} S\right) 4 s 4 p\left({ }^{3} y P\right){ }^{6} P_{7 / 2}$ & 1608.450 & $1608.45085(7)$ & [28-31] \\
\hline $3 d^{5}\left({ }^{6} S\right) 4 s 4 p\left({ }^{1} x P\right){ }^{6} P_{7 / 2}$ & 1260.533 & $1260.53558(6)$ & [28-31] \\
\hline $3 d^{5}\left({ }^{4} G\right) 4 s 4 p\left({ }^{3} y P\right)^{6} F_{11 /}$ & 1144.939 & 1144.93921(5) & [28-31] \\
\hline $3 d^{5}\left({ }^{4} G\right) 4 s 4 p\left({ }^{3} y P\right)^{6} F_{9 / 2}$ & 1143.224 & $1143.22379(6)$ & [28-31] \\
\hline $3 d^{5}\left({ }^{4} G\right) 4 s 4 p\left({ }^{3} y P\right){ }^{6} F_{7 / 2}$ & 1142.366 & $1142.36568(6)$ & [28-31] \\
\hline $3 d^{5}\left({ }^{4} \mathrm{P}\right) 4 \mathrm{~s} 4 \mathrm{p}\left({ }^{3} \mathrm{P}\right)^{6} \mathrm{D}_{7 / 2}$ & 1260.533 & $1133.66526(5)$ & {$[28-31]$} \\
\hline $3 d^{6}\left({ }^{5} D\right) 5 p^{6} D_{9 / 2}$ & 1127.097 & $1127.09842(5)$ & [28-32] \\
\hline $3 d^{6}\left({ }^{5} D\right) 5 p^{6} D_{7 / 2}$ & 1125.446 & 1125.44765(5) & [28-32] \\
\hline $3 d^{5}\left({ }^{4} \mathrm{P}\right) 4 \mathrm{~s} 4 \mathrm{p}\left({ }^{3} \mathrm{P}\right){ }^{6} \mathrm{P}_{7 / 2}$ & 1121.975 & $1121.97473(5)$ & [28-32] \\
\hline $3 d^{6}\left({ }^{5} D\right) 5 p^{6} F_{11 / 2}$ & 1112.047 & $1112.04833(5)$ & [28-32] \\
\hline $3 d^{6}\left({ }^{5} D\right) 5 p^{4} F_{9 / 2}$ & 1106.362 & $1106.35952(5)$ & [28-32] \\
\hline $3 d^{6}\left({ }^{5} D\right) 5 p^{6} P_{7 / 2}$ & 1096.877 & 1096.87689(5) & [29-32] \\
\hline $3 d^{5}\left({ }^{4} \mathrm{D}\right) 4 \mathrm{~s} 4 \mathrm{p}\left({ }^{3} \mathrm{P}\right)^{6} \mathrm{~F}_{9 / 2}$ & 1083.419 & $1083.42027(5)$ & [29-32] \\
\hline $3 d^{5}\left({ }^{4} D\right) 4 s 4 p\left({ }^{3} P\right){ }^{6} F_{11 / 2}$ & 1081.874 & $1081.87528(5)$ & [29-32] \\
\hline $3 d^{5}\left({ }^{4} D\right) 4 s 4 p\left({ }^{3} P\right){ }^{6} D_{7 / 2}$ & 1063.971 & 1063.97182(5) & [29-32] \\
\hline $3 d^{5}\left({ }^{4} D\right) 4 s 4 p\left({ }^{3} P\right)^{6} D_{9 / 2}$ & 1063.176 & 1063.17688(5) & [29-32] \\
\hline $3 d^{5}\left({ }^{4} \mathrm{G}\right) 4 \mathrm{~s} 4 \mathrm{p}\left({ }^{3} \mathrm{wP}\right)^{4} \mathrm{G}_{9 / 2}$ & 1062.152 & 1062.1533(3) & [29-32] \\
\hline $3 d^{5}\left({ }^{4} D\right) 4 s 4 p\left({ }^{3} P\right){ }^{6} P_{7 / 2}$ & 1055.262 & 1055.26178(5) & [29-32] \\
\hline
\end{tabular}

To identify $\Delta \alpha / \alpha$ in each QSO absorber, we used and developed our previous studies with the nonlinear least squares program [28-33]. This program allowed us to fit all available observed absorption line profiles. It is well known that in the case of occurring absorption lines that are available in a single cloud of gas, which does not include the internal velocity structure, these lines would comprise only a single, symmetric component, which will help us make the measurement easier. However, almost all QSO absorption systems have a complicated velocity structure. Therefore,a well-fitting program is needed in order to identify the value of $\Delta \alpha / \alpha$ for these complicated profiles with a minimum number of parameters. This program was developed in order to fit all available transitions simultaneously. Based on the previous studies, we can fit $\Delta \alpha / \alpha$ as a free parameter in our fitting program. Simultaneously, we can estimate the value of $\Delta \alpha / \alpha$ to an associated error for each QSO absorber for the free parameters minimizing $\chi^{2}$. It is noted that the error in the calculated value $\Delta \alpha / \alpha$ is dominated by the error in the measurement of wavelength separation $\Delta \lambda$. The selection of appropriate centroid wavelengths was based on this profile, where the line fitting provides wavelength uncertainties of $\sigma_{\lambda_{1}}$ and $\sigma_{\lambda_{2}}$. In our previous study, we identified that the main effect on the error budget was based on the width separation ratio between observed lines from quasars and laboratories with the actual wavelength splitting of the line pairs with small separations.Therefore, we chose the $\mathrm{Fe}$ 
II lines ( $\lambda 1055-\lambda 2600)$ for our analysis because these lines are located close to the central regions of the corresponding Echelle. Moreover, we have the potential to minimize distortions of the line profiles, which are caused by the decreasing spectral sensitivity at the edges of Echelle orders. In this case, we can distinguish the influence of hidden blends on the line position measurements that help us to identify the identical velocity structure of these transitions. In addition, $\alpha$-independent line ratios are included, which have the benefit of being able to identify the exact size of statistical and systematic errors. In addition to determining the calibrated laboratory wavelength and observed data, issues with the MM technique may be the main cause of potential sources of systematic error. The most important systematic errors and wavelength calibrations affected $\Delta \alpha / \alpha$ determinations in the previous studies. Because these effects are dependent on the transition wavelength therefore, they have to be quantified and removed. We know that there are common systematic errors, such as the incorrect wavelength solution through the thorium-argon (ThAr) calibration. Moreover, these errors can result from velocity shifts between spectra from different exposures, and from different settings on the spectrograph, which the MM method is unable to explain. Furthermore, one of the most important problems is these systematic errors themselves, which might introduce a velocity distortion as opposed to a simple velocity offset. It is well known that the obtained results for $\Delta \alpha / \alpha$ measurement would not be correct if there was a velocity distortion inside the spectrum, because the MM technique is sensitive to velocity shifts between transitions. The disadvantage of the MM method is the insufficient spectral resolution coming from the present QSO spectra. At present, we can only detect about $60 \%$ of the individual subcomponents that come from galactic interstellar absorption lines with very high-resolution spectra $\left(R=10^{6}\right)$. The normal separation of these subcomponents is approximately 1.2 $\mathrm{km} \mathrm{s}^{-1}$ even at a resolution of $0.5 \mathrm{~km} \mathrm{~s}^{-1}$ [34-39]. It should be noted that the Doppler profiles could possibly narrow in the highest-quality QSO spectra $\left(R \approx 80,000 \sim 4 \mathrm{~km} \mathrm{~s}^{-1}\right)$, in which the saturated subcomponents could be recognized only by line asymmetries. This problem has been identified in the works of Murphy et al. [38] and their analysis focused on possible weak transition lines and therefore, the effects of which were statistically relevant for a large number of systems. The results found by Chand et al., which included closely blended components, probed $\Delta \alpha / \alpha$ regarding the apparent position shifts caused by unresolved line blends [39]. The results came from different studies based on their type of analysis, therefore they restricted their work to systems with simple profiles. One must be aware that within their works there have been reported calibration errors that are much larger than the final velocity precision required to determine $\Delta \alpha / \alpha$. These calibration errors are possibly averaged out and they do not support a good method for the final determination $\Delta \alpha / \alpha$. In the case of the MM method, the comparison of various lines with various absorbers has been found in a large number of redshifts. If these errors are random, they may average out. However, these errors will not affect our rate changes in measured Doppler shifts with redshift $z$. Making use of this technique to find these errors, we first estimated $\Delta \alpha / \alpha$ for a single line of observed spectrum, then we applied this to the whole spectrum to determine the average error of all systems.

\section{Results and Discussion}

In our previous works, the best result obtained was the variation limits of $\Delta \alpha / \alpha=2.2 \pm 2.3 \mathrm{ppm}$ (parts per million). However, these works suggested that this limit was caused by a systematic effect, which can be determined from the use of laboratory wavelengths. We have since improved these conditions by refining our previous method using wavelength shifts in combination with the laboratory Ritz wavelengths and QSO spectra with anuncertainty of $0.004 \AA$. This allowed us to examine the suspected systematic errors, which may be caused by the calibration error. Moreover, this effect has important implications, since it is dependent on the wavelength transitions used to determine the value of $\Delta \alpha / \alpha$. The spectral data were first examinedandassociated with every object, then the absorption lines were fitted to determine the Fe II transitions in order to estimate the relevant values of $\Delta \alpha / \alpha$. Blends of wavelength were inspected for all transitions and discarded, if found. We fit the Fe II absorption lines in this way using previously-described method [28-33]. For each individual system 
variation of $\alpha$, we utilized the relative positions of whole lines, instead of comparing the positions of single components in the fitting parameters (column density $\mathrm{N}$, the absorption redshift, the Doppler, and line width $b$ ) between the components, which were assumed to be the same as we expected for all transitions. The velocity or redshift scale was calculated to identify the positions ofthe Fe II transitions thatwere applied in our profile analysis. Often, $\alpha$ was kept as an additional parameter for our fitting procedure. Inthis way, we coulddetermine any change in $\alpha$ based on the determination of the shifted transitions on each line of gas. Based on the previous fitting program, we used this profile in accordance with the reduced variation of $\chi^{2}$ as a general function of $\Delta \alpha / \alpha$ from each absorber fitting. This program included a set of fixed values and free parameters thatdescribe the atomic and molecular properties together with those absorbing systems.The fitting procedure of Fe II was based on the set ofincluded parameters: The column density $\mathrm{N}$, the absorption redshift $z_{a b s}$, together with the Doppler and line-width $\mathrm{b}$. Based on these varying parameters, we varied $\Delta \alpha / \alpha$ starting from $-1.2 \times 10^{-5}$ to $1.2 \times 10^{-5}$ in a step of $-0.1 \times 10^{-5}$ for all those lines. In this case, we accepted only $\chi^{2}$ minimum $\left(\chi_{\min }^{2}\right)$ as it was the measured valuation of $\Delta \alpha / \alpha$ with the system. Then we retrieved the minima $\chi^{2}$ for each of these fits and plotted them as a general function of $\Delta \alpha / \alpha$. Therefore, to make sure that the minima $\chi^{2}$ regarding our crucial parameter was not a local minimum, we made use of the contrary $\chi^{2}$ to the $\Delta \alpha / \alpha$ curvature method as opposed to a single minimization that we could concurrently base the variation of four parameters; $\Delta \alpha / \alpha$, Doppler, widths, and redshifts. Despite what might be expected, we minimized $\chi^{2}$ by various Doppler widths and redshifts for each valuation of $\Delta \alpha / \alpha$. We then determined the minima of $\chi^{2}$ versus $\Delta \alpha / \alpha$ and all Fe II lines wereapplied to fit simultaneously using laboratory wavelengths $(\Delta \alpha / \alpha=0)$. Therefore, the parameter of redshifts was constrained in this way so thatall species under consideration would be the same. We minimized $\chi^{2}$ using our fit and estimated the final value of $\Delta \alpha / \alpha$ with its statistical error correspondence and the systematic error with $1 \sigma$ uncertainty together with the smaller reduced $\chi^{2}$ in Table 2 .

Step by step, this fitting program was used to recover the value of $\Delta \alpha / \alpha$ in each realization and it was plotted as a function of the input. In an individual measurement of the typical error, $\Delta \alpha / \alpha$ was also illustrated in the plot. Consequently, we had 27 input values with included varying parameters: Column density $\mathrm{N}, z_{a b s}$ Doppler, and line-width $\mathrm{b}$. Therefore, each point shows the location of the minimum in $\Delta \alpha / \alpha$ with an error bar $1 \sigma$. The output values of $\Delta \alpha / \alpha$ are represented by the weighted mean of the error bars from 27 measurements, which are consistent with the input values for each absorption system, as illustrated in Figure 1. We obtained the final result: $\Delta \alpha / \alpha=$ $(0.027 \pm 0.832) \times 10^{-6}$, where the error was determined with a high degree of accuracy.

In the analysis of the optical quasar spectra, the works of Quast et al. (2004); Chand et al. (2006); Levshakov et al. $(2006 ; 2007 ; 2008)$ and Murphy et al. (2008) are conflicting, at least in part $[15-19,27]$. These analyses of $\Delta \alpha / \alpha$ was certainly not explicitly used as the fitting parameter. Instead, they used the $\chi^{2}$ versus $\Delta \alpha / \alpha$ curve to acquire the best-fitting value of $\Delta \alpha / \alpha$. The most effective result of those analyses only agreed with a cosmological variation of the level of $10^{-6}$. To search for the sensitivity over cosmic space and time, our analyses were based on the $\alpha$-independent line ratios with the main influence range on the error budget. This allowed us to perceive the actual size of statistical and systematic errors and trust the exact wavelength splitting of the line pairs, which was also mostly suggested around the lines with small separations. Moreover, it was simply and well-applied to a wide redshift range, leading to the final possibility to imply that regardless of the averaging over $\Delta \alpha / \alpha$, several values will deliver an accurate $\Delta \alpha / \alpha$ value. 
Table 2. Redshift order of $\Delta \alpha / \alpha$ was calculated and combined the QSO and Ritz wavelengths for Fe II and the weighted average of all lines. Based on the standard statistical process, we assigned a $1 \sigma$ error within the above and outlined the best value of $\Delta \alpha / \alpha$ in order for $\Delta \chi^{2}=\chi^{2}-\chi_{\min }^{2}=1$ to be computed for the required change in $\Delta \alpha / \alpha$. The maximum change in $\Delta \alpha / \alpha$ was then determined, such as $\Delta \chi^{2}=1$ in the estimation error of $\Delta \alpha / \alpha$. Then we determined the minima $\chi^{2}$ for each of these fits and plotted these as a general function of $\Delta \alpha / \alpha$. The expected result was $\Delta \alpha / \alpha=(0.027 \pm 0.832) \times 10^{-6}$ with the standard deviation of error around the weighted mean $\left(\sigma_{t o t}^{2}=\sigma_{\Delta \alpha / \alpha}^{2}+\sigma_{s y s}^{2}\right)$.

\begin{tabular}{|c|c|c|}
\hline$z\left(10^{-6}\right)$ & $\Delta \alpha / \alpha\left(10^{-6}\right)$ & $\sigma_{\Delta \alpha / \alpha}\left(10^{-6}\right)$ \\
\hline 0.05 & -0.0194 & 0.49997 \\
\hline 0.04197 & 0.05036 & 0.41968 \\
\hline 0.05876 & -0.01898 & 0.58755 \\
\hline 0.02106 & -0.27398 & 0.21057 \\
\hline 0.52374 & 0.2802 & 0.52374 \\
\hline 0.02559 & 0.04099 & 0.25595 \\
\hline 0.10173 & 3.43353 & 1.01735 \\
\hline 2.80641 & -2.23371 & 2.80641 \\
\hline 0.39101 & -0.20865 & 0.39101 \\
\hline 0.02487 & 0.01243 & 0.24869 \\
\hline 2.04675 & 0.62672 & 2.04675 \\
\hline 0.18342 & 0.19726 & 1.83416 \\
\hline 0.20993 & 0.03515 & 2.09932 \\
\hline 0.28012 & -0.25867 & 2.8012 \\
\hline 0.22934 & 0.14743 & 2.29345 \\
\hline 0.06211 & -0.00441 & 0.62106 \\
\hline 0.05331 & 0.09425 & 0.53312 \\
\hline 0.24065 & -0.09503 & 2.40647 \\
\hline 0.05395 & 1.10512 & 0.53954 \\
\hline 2.24159 & -1.08932 & 2.24159 \\
\hline 0.10028 & -0.17914 & 1.00285 \\
\hline 0.24921 & -0.00481 & 2.49211 \\
\hline 0.25881 & 0.22132 & 2.5881 \\
\hline 0.16918 & -0.49919 & 1.69177 \\
\hline 0.82771 & -0.19849 & 0.82771 \\
\hline 1.22393 & 0.72562 & 1.22393 \\
\hline 0.20848 & 1.03206 & 0.20848 \\
\hline
\end{tabular}

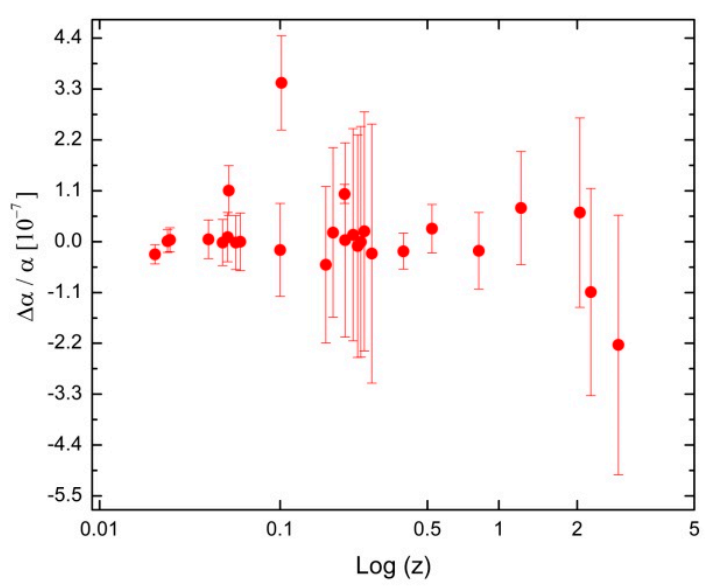

Figure 1. The values of $\Delta \alpha / \alpha$ are illustrated versus redshift for the Fe II absorption lines. The estimated values of $\Delta \alpha / \alpha$ from our analysis are plotted based on the $\chi^{2}$ minimization for individual systems.

\section{Conclusions}

In this study, we used Ritz wavelengths, including 27 lines of Fe II to constrain past variations of $\alpha$. The expected result was probably the most stringent limit on the $\alpha$-variation of an individual 
absorption line. Moreover, this result indicates that the data-reduction, calibration, and analysis of Fe II systems eliminated the most important systematic errors associated with other determinations of $\alpha$ and offered estimations of the remainder of the statistical and systematic errors. This arose because our analysis only included $\alpha$-independent line ratios which can identify the real size of statistical and systematic errors.

The obtained values of $\Delta \alpha / \alpha$ from our analysis were plotted versus the absorption redshifts of Fe II systems. Despite the low-z data, we found on of the most stringent limits on the space and time variations in $\Delta \alpha / \alpha$. The large scatters seen in $\Delta \alpha / \alpha$ for the high-z systems occurred mainly because of the large change in the Fe II wavelengths. Furthermore, the values of $\chi^{2}$ indicated that a linear increase in $\alpha$ with time was preferred. As a result, whenever we look deep into the early universe (high redshift), the values of $\Delta \alpha / \alpha$ are positive or negative as a result of the systematic error effect that is based on the use of the laboratory wavelengths.

The precision regarding the relative change in aachieved from our results is the most stringent limit in comparison with the results produced by Quast et al. (2004) [15]. Our results also possess order of magnitude improvements compared to the exact results from Chand et al. (2006) [16] and are better than the results derived by Levshakov et al. (2006; 2007; 2008) [17-19], Molaro et al. (2005; 2008) [14-25], Murphy et al. (2008; 2014) [27,34], and Bainbridge et al. (2017) [40]. A considerable advance has been made in improving the laboratory wavelengths of transitions in the Fe II multiplet line that can be utilized in our analyses. These remarks will provide the strongest and most constraining measurements, with particular implications for the cosmic space-time variation of the fine-structure constant, allowing further investigation of large systems in order to reduce the final error bar [11-13,34-42]. This result creates a better choice with a high-quality process for the selection of the absorption lines. Moreover, it would allow us to reduce the potential for systematic error effects inthe estimation of the $\Delta \alpha / \alpha$ values of the quality of many multiplet systems available in a single QSO spectrum with high sensitivity.

With the further development of QSO as well as the improved laboratory wavelengths, this study ought additionally to be able to open an innovative window for the equivalence principle of relativity and can provide an important tool for checking the models of the grand unification.

Funding: This research received no external funding.

Conflicts of Interest: The authors declare no conflict of interest.

\section{References}

1. Dirac, P.A.M. The cosmological constants. Nature 1937, 139, 323. [CrossRef]

2. Milne, E.A. Kinematics, dynamics, and the scale of time. Proc. R. Soc. A 1937, 158, 324-348. [CrossRef]

3. Savedoff, M.D. Physical constants in extra-galactic nebulae. Nature 1995, 178, 688-689. [CrossRef]

4. Bahcall, J.N.; Schmidt, M. Does the fine-structure constant vary with cosmic time? Phys. Rev. Lett. 1967, 19, 1294-1295. [CrossRef]

5. Bahcall, J.N.; Steinhardt, C.L.; Schlegel, D. Does the fine-structure constant vary with cosmological epoch? Astrophys. J. 2004, 600, 520-543. [CrossRef]

6. Grupe, D.; Pradhan, A.; Frank, S. Studying the variation of the fine-structure constant using emission-line multiplets. Astron. J. 2005, 130, 355-366. [CrossRef]

7. Dzuba, V.A.; Flambaum, V.V.; Webb, J.K. Calculations of the relativistic effects in many-electron atoms and space-time variation of fundamental constants. Phy. Rev. A. 1999, 59, 230-237. [CrossRef]

8. Webb, J.K.; Flambaum, V.V.; Christopher, W.; Drinkwater, M.J.; Barrow, J.D. Search for time variation of the fine structure constant. Phy. Rev. Lett. 1999, 82, 888-891. [CrossRef]

9. Dzuba, V.A.; Safronova, U.I.; Johnson, W.R. Energy levels and lifetimes of Nd IV, Pm IV, Sm IV, and Eu IV. Phys. Rev. A 2003, 68, 032503. [CrossRef]

10. Webb, J.K.; Murphy, M.T.; Flambaum, V.V.; Dzuba, V.A.; Barrow, J.K.; Churchill, C.W.; Prochaska, J.X.; Wolfe, A.M. Further evidence for cosmological evolution of the fine structure constant. Phys. Rev. Lett. 2001, 87, 091301. [CrossRef] 
11. Murphy, M.T.; Webb, J.K.; Flambaum, V.V. Further evidence for a variable fine structure constant from Keck/HIRES QSO absorption spectra. Mon. Not. R. Soc. 2003, 245, 609-638. [CrossRef]

12. Murphy, M.T.; Webb, J.K.; Flambaum, V.V.; Curran, S.J. Time evolution of the fine structure constant. arXiv 2002, arXiv:astro-ph/0209488.

13. Webb, J.K.; Murphy, M.T.; Flambaum, V.V.; Curran, S.J. Does the fine structure constant vary? A third quasar absorption sample consistent with varying $\alpha$. Astrophys. Space Sci. 2003, 283, 565-575. [CrossRef]

14. Reimers, D.; Hagen, H.; Rodriguez, P.; Wisotzki, L. Detection of further UV-bright high-redshift QSOs. Astron. Astrophys. 1998, 334, 96-98.

15. Quast, R.; Reimers, D.; Levshakov, S.A. Probing the variability of the fine-structure constant with the VLT/UVES. Astron. Astrophys. 2004, 415, L7-L11. [CrossRef]

16. Chand, H.; Srianand, R.; Petitjean, P.; Aracil, B.; Quast, R.; Reimers, D. Variation of the fine-structure constant: Very high resolution spectrum of QSO HE 0515-4414. Astron. Astrophys. 2006, 451, 45-56. [CrossRef]

17. Levshakov, S.A.; Centurión, M.; D’Odorico, S.; Reimers, D.; Quast, R.; Pollmann, M. Most precise single redshift bound to $\Delta \alpha / \alpha$. Astron. Astrophys. 2006, 449, 879-889. [CrossRef]

18. Levshakov, S.A.; Molaro, P.; Lopez, S.; D’Odorico, S.; Centurión, M.; Bonifacio, P.; Agafonova, I.I.; Reimers, D. A new measure of $\Delta \alpha / \alpha$ at redshift $z=1.84$ from very high resolution spectra of Q 1101-264. Astron. Astrophys. 2007, 446, 1077-1082. [CrossRef]

19. Levshakov, S.A.; Reimers, D.; Kozlov, M.G.; Porsev, S.G.; Molaro, P. A new approach for testing variations of fundamental constants over cosmic epochs using FIR fine-structure lines. Astron. Astrophys. 2008, 479, 719-723. [CrossRef]

20. Porsev, S.G.; Koshelev, K.V.; Tupitsyn, I.I.; Kozlov, M.G.; Reimers, D.; Levshakov, S.A. Transition frequency shifts with fine-structure-constant variation for Fe II: Breit and core-valence correlation corrections. Phys. Rev. A 2007, 76, 052507. [CrossRef]

21. Agafonova, I.I.; Molaro, P.; Levshakov, S.A.; Hou, J.L. First measurement of Mg isotope abundances at high redshifts and accurate estimate of $\Delta \alpha / \alpha$. Astron. Astrophys. 2011, A28, 529. [CrossRef]

22. Griest, K.; Whitmore, J.B.; Wolfe, M.; Prochaska, J.X.; Howk, J.C.; Marcy, G.W. Wavelength Accuracy of the Keck Hires Spectrograph and Measuring Changes in the Fine Structure Constant. Astrophys. J. 2010, 708, 158-170. [CrossRef]

23. Martinez, A.F.; Vladiloand, F.G.; Bonifacio, P. Search for alpha variation in UVES spectra: Analysis of C IV and Si IV doublets towards QSO 1101-264. arXiv, 2003; arXiv:astro-ph/0312270.

24. Levshakov, S.A.; Centurión, M.; Molaro, P.; D'Odorico, S. VLT/UVES constraints on the cosmological variability of the fine-structure constant. Astron. Astrophys. 2005, 434, 827-838. [CrossRef]

25. Molaro, P.; Levshakov, S.A.; Monsai, S.; Centurión, M.; Bonifacio, S.; D’Odorico, S.; Monaco, L. UVES radial velocity accuracy from asteroid observations I. Implications for fine structure constant variability. Astron. Astrophys. 2008, 481, 559-569. [CrossRef]

26. Gutierrez, C.M.; Lopez-Corredoira, M. The value of the fine structure constant over cosmological times. Astrophys. J. 2010, 713, 46-51. [CrossRef]

27. Murphy, M.T.; Flambaum, V.V.; Muller, S.; Henkel, C. Strong limit on a variable proton-to-electron mass ratio from molecules in the distant universe. Science 2008, 320, 16111613. [CrossRef] [PubMed]

28. Le, T.D. A Stringent Limit on Variation of the Fine-Structure Constant Using Absorption Line Multiplets in the Early Universe. Astrophys. 2016, 59, 285-291. [CrossRef]

29. Aldenius, M.; Johansson, S.; Murphy, M.T. Accurate laboratory ultraviolet wavelengths for quasar absorption-line constraints on varying fundamental constants. Mon. Not. R. Soc. 2006, 370, 444-452. [CrossRef]

30. Nave, G.; Sansonetti, C.J. Wavelengths of the $3 d^{6}\left({ }^{5} \mathrm{D}\right) 4 \mathrm{~s} \mathrm{a}^{6} \mathrm{D}-3 \mathrm{~d}^{6}\left({ }^{5} \mathrm{D}\right) 4 \mathrm{py} \mathrm{y}^{6} \mathrm{P}$ multiplet of Fe II (UV 8). Opt. Soc. Am. 2011, B28, 737-745. [CrossRef]

31. Nave, G. Wavelengths of Fe II lines for studies of time variation of the fine-structure constant. Mon. Not. R. Soc. 2012, 420, 1570-1574. [CrossRef]

32. Pickering, J.C.; Thorne, A.P.; Murray, J.E.; Litzén, U.; Johansson, S. Accurate laboratory wavelengths of some ultraviolet lines of $\mathrm{Cr}, \mathrm{Zn}$ and Ni relevant to time variations of the fine structure constant. Mon. Not. R. Soc. 2000, 319, 163-167. [CrossRef] 
33. Pickering, J.C.; Donnelly, M.; Nilsson, H.; Hibbert, A.; Johansson, S. The FERRUM Project: Experimental oscillator strengths of the UV 8 multiplet and other UV transitions from the $\mathrm{y}^{6} \mathrm{P}$ levels of Fe II. Astron. Astrophys. 2002, 396, 715-722. [CrossRef]

34. Murphy, M.T.; Berengut, J.C. Laboratory atomic transition data for precise optical quasar absorption spectroscopy. Mon. Not. R. Soc. 2014, 438, 388-411. [CrossRef]

35. Welty, D.E.; Hobbs, L.M.; Kulkarni, V.P. A high-resolution survey of interstellar NA I D1 lines. Astrophys. J. 1994, 436, 152-175. [CrossRef]

36. Welty, D.E.; Morton, D.C.; Hobbs, L.M. A High-Resolution Survey of Interstellar Ca II Absorption. Astrophys. J. 1996, 106, 533-562. [CrossRef]

37. Wendt, M.; Molaro, P. Robust limit on a varying proton-to-electron mass ratio from a single $\mathrm{H}_{2}$ system. Astron. Astrophys. 2011, A96, 526.

38. Murphy, M.T.; Webb, J.K.; Flambaum, V.V.; Dzuba, V.A.; Churchill, C.W.; Prochaska, J.X. Possible evidence for a variable fine-structure constant from QSO absorption lines: Motivations, analysis and results. Mon. Not. R. Soc. 2001, 327, 1223-1236. [CrossRef]

39. Chand, H.; Srianand, R.; Petitjean, P.; Aracil, B. Probing the cosmological variation of the fine-structure constant: Results based on VLT-UVES sample. Astron. Astrophys. 2004, 417, 853-871. [CrossRef]

40. Bainbridge, M.B.; Webb, J.K. Artificial intelligence applied to the automatic analysis of absorption spectra.Objective measurement of the fine structure constant. Mon. Not. R. Soc. 2017, 468, 1639-1670.

41. Webb, J.K.; King, J.A.; Murphy, M.T.; Flambaum, V.V.; Carswell, R.F.; Bainbridge, M.B. Indications of a Spatial Variation of the Fine Structure Constant. Phys. Rev. Lett. 2011, 107, 191101. [CrossRef]

42. Bagdonaite, J.; Jansen, P.; Henkel, C.; Bethlem, H.L.; Menten, K.M.; Ubachs, W. A stringent limit on a drifting proton-to-electron mass ratio from alcohol in the early universe. Science 2012, 339, 46-48. [CrossRef]

(C) 2018 by the author. Licensee MDPI, Basel, Switzerland. This article is an open access article distributed under the terms and conditions of the Creative Commons Attribution (CC BY) license (http:/ / creativecommons.org/licenses/by/4.0/). 